\title{
The model of the closed Universe with relativistic effects of extra dimension
}

\author{
Zahid Zakir ${ }^{1}$
}

\begin{abstract}
In the standard general relativity (GR) a closed universe as a 3 -sphere exists only at embedding into a real 4-space and a hypersurface of simultaneity of the universe is spherically-symmetric only in a global static frame where the center of the 3 -sphere is rested. For this reason the evolution of the universe is described in a world time of this frame, where Einstein's equations with homogeneous matter (without dark energy) have simple exact solutions. The radial speed of 3 -sphere leads to the relativistic delay of proper times, which then leads to new expansion law and relativistic redshift. By using the initial data - present radius and velocity of expansion - other properties and evolution of the Universe are predicted and are in agreement with the data. As the result, from standard GR and observations follow that the Universe is closed, slowing down and the extra dimension probably really exist. The evolution is described for all epochs by taking into account pressure in earlier epochs. The nucleosynthesis and recombination occur at sufficiently smaller concentrations, but during sufficienly longer times. It is shown that the model is free on the cosmological problems of former approaches.
\end{abstract}

PACS: 04.20.Cv, 98.80.-k, 98.80.Jk 95.30.Sf, 97.60.Lf, 98.35.Jk, 98.54.-h, 98.80.-k, 04.60.-m Key words: cosmological models, redshift, supernovae, Big Bang, nucleosynthesis, recombination, cosmic microwave background, cosmological problems

\section{Content}

Introduction 38

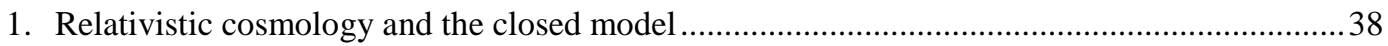

1.1. Stages of development of the closed model ..................................................................... 38

1.2. The closed model with the relativistic time dilation ..........................................................4 40

1.3. The relativistic redshift and parameters of the Universe ..................................................4

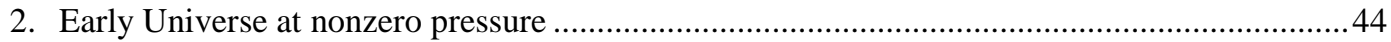

2.1. Closed universe in 4-space at nonzero pressure ............................................................4

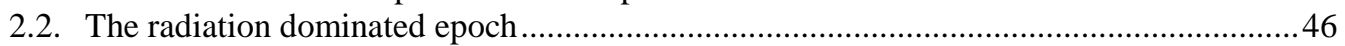

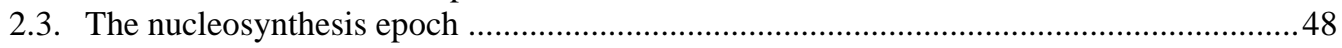

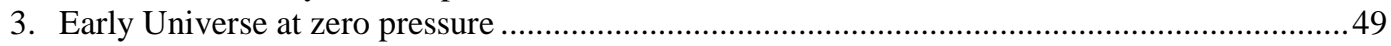

3.1. Expansion and contraction of a thin dust 3 -sphere in 4 -space ........................................49

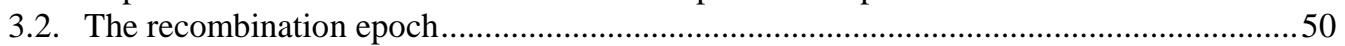

4. The life cycle of the Universe and cosmological problems ....................................................51

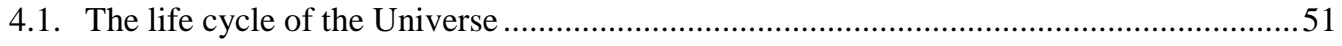

4.2. The lack of former main cosmological problems........................................................5

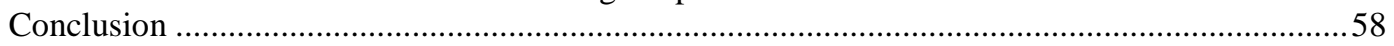

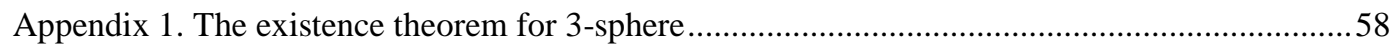

Appendix 1. Einstein's equations for the closed model in terms of world time .............................59

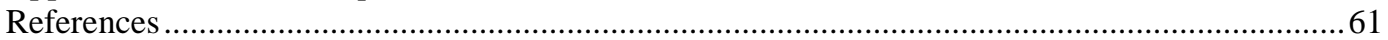

${ }^{1}$ Centre for Theoretical Physics and Astrophyics, Tashkent, Uzbekistan; zahidzakir@theor-phys.org 


\section{Introduction}

From three of possible models of relativistic cosmology for homogeneous and isotropic universe - closed, flat and open - the closed model is preferable from theoretical point of view since only in it 3-volume and mass of the universe are finite and the fact of Big Bang follows from it naturally without additional hypotheses and boundary conditions in 3-space [1-7]

In the previous paper [8] a model of closed universe with relativistic redshift has been formulated as simplest consistent model of relativistic cosmology. It has been shown that the model is parameter free and by using only initial data for the universe - the present day radius $a_{0}$ and expansion velocity $\dot{a}_{0}$ - well describes observational data on the cosmological redshift and the matter density, predicts other properties and evolution history of the universe.

In the present paper the model is formulated for all epochs, including the early universe. It is shown that at taking into account of pressure with the standard equations of state the model remains to be simple and exactly solvable. This allows one to study evolution of the universe at practically all epochs on the basis of quite simple relationships.

Particularly, the modified physical conditions and parameters of the nucleosynthesis and recombination are discussed. It is shown that the model allows one to describe the observational data without new hypotheses and arbitrary parameters. The same model, but without the relativistic redshift and on the basis of the dark energy, has been studied in [9].

In the first part of the paper the closed model in 4-space is formulated in simplest case of a pressureless matter. Other epochs, including the epochs of recombination, nucleosynthesis and earlier universe with nonzero pressure, and also solutions of main cosmological problems are considered in the sections 2-4.

\section{Relativistic cosmology and the closed model}

\subsection{Stages of development of the closed model}

After the formulation of GR, its first application to cosmology by Einstein [1] has shown in principle new opportunity to dispense with boundary conditions in 3 -space, by eliminating a set of contradictions, - the universe could be a 3-sphere of finite volume containing finite mass (energy). He started by a linear element in 3-space which in spherical coordinates has the form:

$$
d s^{2}=d \tau^{2}-a^{2}(\tau) d^{2} \Omega_{3},
$$

where $\tau$ is the proper time and $d^{2} \Omega_{3}=d \chi^{2}+\sin ^{2} \chi \cdot d^{2} \Omega_{2}$ is an element of angular distance on 3-sphere. Einstein also has based on two hypotheses - about 3-dimensionality of universe's space and its static evolution, but since the 3-sphere's radius $a(\tau)$ changes in time, he added third hypothesis about a stabilizing cosmological constant $\Lambda \neq 0$.

After shoving the lack of stable static solutions and developing of non-static world models [2], the second hypothesis has been rejected, which then had make as optional the third one. Then the evolution equation has taken the form:

$$
\left(\frac{d a}{d \tau}\right)^{2}=\frac{a_{m}}{a}-1+\Lambda a^{2} .
$$


Discovery of redshift $z_{s}$ in spectra of galaxies, defined as a relative increasing of wavelength $z_{s} \equiv \Delta \lambda / \lambda_{e}$, i.e. $\lambda_{r} / \lambda_{e} \equiv 1+z_{s}$, has shown the expansion of the Universe $[3,4]$. Spectroscopic redshift $z_{s}$ was thus equated to a relative increasing of radius $z \equiv \Delta a / a_{z}$, i.e. $z_{s}=z$. Thus, here

$$
a_{0} / a_{z} \equiv 1+z
$$

From the fact of the expansion it was followed a conclusion that an initial state of the Universe was very dense and thus very hot, which led to the present expansion [6]. Later this picture formed a basis of the Big Bang theory [7] which explained abundance of light elements and predicted the cosmic microwave background (CMB).

The formulation of models based on Eqs.(1)-(3), a derivation of Eq.(2) from dynamics of a ball in homogeneous world [5], observational improvements of the parameters of "luminosity-redshift" relation, matter density and discovery of CMB have ended the initial stage of formation of relativistic cosmology.

Main problems of the second stage were: 1) improvement of distances and luminosities for distant sources; 2) definition of curvature and choice of a cosmological model; 3) construction and checking of scenarios of the early and future Universe; 4) construction and checking of scenarios of structure formation. Further the development of theory in first three directions will be considered.

Last decades Type 1a supernovae (SN 1a), as "standard candles", have shown that objects with increasing of $z$ become dimmer [10] than it follows from Eqs.(1)-(3). This fact has been tried to explain in models with $\Lambda \neq 0$ and flat metric, but as before being based on Eqs.(1)-(3), by believing to their correctness in standard GR.

But, the evolution equation (2) leads to an interior contradiction in the theory since it follows from the nonrelativistic dynamics of a ball [5], where it did not taken into account relativistic kinematics essentially modifying the evolution equation at large radii. The extrapolation of nonrelativistic dynamics of the ball to entire Universe is obviously incorrect and testifies that Eqs.(1)-(3) should be somehow modified.

Other interior inconsistency in the former closed models followed from the fact [11] that the existence of a 3-sphere is impossible without embedding it into at least 4space (see Appendix 1). Therefore, the formulation of the closed models in 3-space and their "confrontation" with observations also were incorrect initially.

Attempts to modify the closed model, with taking into account the relativistic time dilation in terms of world time $t$ of rest frame of 3-sphere's centre in real 4-space, have been attempted by many authors. In simplest kinematic formulation [9] the proper time has been introduced as:

$$
d \tau^{2}=d t^{2}\left(1-\dot{a}^{2}\right)
$$

The linear element then transfers into

$$
d s^{2}=d t^{2}\left(1-\dot{a}^{2}\right)-a^{2}(t) d^{2} \Omega_{3},
$$

and the evolution equation has the form

$$
\frac{\dot{a}^{2}}{1-\dot{a}^{2}}=\frac{a_{m}}{a}-1+\Lambda a^{2} .
$$

But, in [9], by modifying Eqs. (1)-(2), it has been preserved Eq. (3) and, for description of observations, the dark energy $\Lambda \neq 0$, determining the dynamics and parameters of the Universe, has been introduced.

The definition (3) has revised firstly only recently [12] when it has been found that in the real Universe GR leads to nontrivial relation of $z_{s}$ with $a$ because of the stasis 
of $z_{s}$ in each of passed by radiation galaxy cluster. As a result, the spectroscopic $z_{s}$ and the cosmological $z$ become unequal $z_{s}<z$, and thus it is necessary always to "improve" the observed $z_{s}$.

The second, more serious revision of (3) has been required because of finding of the relativistic redshift [8] following from (4) at high speed of expansion of ball's surface in the homogeneous world. Then from Eqs.(5)-(6) (at $\Lambda=0$ ) follows $1-\dot{a}^{2}=a / a_{m}$ and in addition to the linear stretching at expansion of wavelengths, as well as periods in world time $\delta t_{r} / \delta t_{e}=a_{0} / a_{z}$, there appears the transverse Doppler-effect too:

$$
\frac{\delta \tau_{r}}{\delta \tau_{e}}=\frac{\delta t_{r} \sqrt{1-\dot{a}_{0}^{2}}}{\delta t_{e} \sqrt{1-\dot{a}_{z}^{2}}}=\frac{a_{0}^{3 / 2}}{a_{z}^{3 / 2}}=1+z
$$

It together with Eqs. (4)-(6) leads to the replacement of (3) by (7) or $a_{0} / a_{z}=(1+z)^{2 / 3}$ and provides the agreement of the theory with observations. Below this version of the closed model with relativistic redshift (and without dark energy) is developed systematically.

\subsection{The closed model with the relativistic time dilation}

The evolution of the closed universe we will consider in $K_{m}$ frame where the centre of 3-sphere is rested and a global hypersurface of simultaneity of the universe is 3sphere. Here we take into account only the attraction of matter in 3-sphere and the relativistic effects due to the speed of expansion.

In this case the coordinate time $t$ of the system $K_{m}$ is cosmological world time which goes uniformly during universe's evolution. There is a set of equidistant hypersurfaces of simultaneity $t=$ const on any of which a 3-spherical spatial physical system of coordinates is constructed. The rate of standard clocks showing proper times, instantly the same anywhere on the 3-sphere, is slowed down differently at different moments of expansion. In contrast, the rate of coordinate clocks, measuring the world time $t$ and going faster with respect to the comoving to the matter standard clocks, is the same at any time of the expansion and all these coordinate clocks remain mutually synchronous.

For the line element on 3-sphere in the rest frame of the sphere $K_{m}$ we have the same expression as for 2-sphere in 3-space, but with increasing to one the dimensionality of the angular linear element only $d^{2} \Omega_{2} \rightarrow d^{2} \Omega_{3}$ :

$$
d s^{2}=d t^{2}-d a^{2}-a^{2}(t) d^{2} \Omega_{3}=d t^{2}\left(1-\dot{a}^{2}\right)-a^{2}(t) d^{2} \Omega_{3} .
$$

Thus, world time $t$ is measured at each point by the coordinate clocks going faster with respect to the standard clocks with the relativistic factor $\left(1-\dot{a}^{2}\right)^{1 / 2}$.

This form reveals an important fact - the line element (8) in $D=1+4$ spacetime is equivalent to a line element in $D=1+3$ spacetime with non-trivial temporal component of the metrics:

$$
d s^{2}=e^{v(t)} d t^{2}-a^{2}(t) d^{2} \Omega_{3} .
$$

As the result, in this approach both representations (8) and (9) are equivalent and the appearance of the temporal metrics

$$
g_{00}(t)=e^{v(t)}=1-\dot{a}^{2}(t)
$$


is related by the kinematical effects - the relativistic time dilation at cosmological expansion which leads to the relativistic Doppler effect.

Thus, for the expression of the expansion speed $\dot{a}(t)$ through the parameters of the model it is enough to solve Einstein's equation $G_{v}^{\mu}=\kappa_{4} T_{v}^{\mu}$ in $D=1+3$ spacetime with the metric (10) (details see in Appendix 2). The Einstein equations (92)-(93) (at $p=0)$ turn to the relations:

$$
\frac{1}{1-\dot{a}^{2}}=\frac{a_{m}}{a}, \quad \rho a^{3}=\text { const } .
$$

where the maximal radius of expansion $a_{m}$ is defined by the condition $\dot{a}=0$ :

$$
a_{m} \equiv \frac{1}{3} \kappa_{4} \rho_{0} a_{0}^{3}=2 G M, \quad \kappa_{4}=12 \pi^{2} G .
$$

For the expansion speed and an expression of the metric (11) across $a$ we find:

$$
\begin{gathered}
e^{v / 2}=\sqrt{1-\dot{a}^{2}}=\sqrt{a / a_{m}} . \\
\dot{a}= \pm \sqrt{1-a / a_{m}}, \quad a(t)=\left|t-t^{2} / 4 a_{m}\right| .
\end{gathered}
$$

The world time from the beginning up to the maximal expansion is $t_{m}=2 a_{m}$. The age of the universe in world time is:

$$
t_{0}=2 a_{m}(1-\sqrt{1-b}), \quad b \equiv a_{0} / a_{m} .
$$

The proper time from the beginning of the dust stage of expansion is:

$$
\tau_{1}=\int_{0}^{t_{1}} d t \sqrt{1-\dot{a}^{2}}=-\int_{0}^{a_{1}} \frac{d a \sqrt{a}}{\sqrt{a_{m}-a}}=a_{m} \arcsin \sqrt{\frac{a_{1}}{a_{m}}}-\sqrt{a_{1}\left(a_{m}-a_{1}\right)},
$$

and for the proper time age of the dust period of evolution we obtain:

$$
\tau_{0}=a_{m}(\arcsin \sqrt{b}-\sqrt{b(1-b)}) \text {. }
$$

The proper time interval from the beginning of (dust period) expansion up to stopping at $a\left(\tau_{m}\right)=a_{m}$, thus, is equal to $\tau_{m}=a_{m} \pi / 2$ and it is less than the coordinate time interval up to this moment: $\tau_{m}<t_{m}$. At early epochs $\left(t \ll a_{m}\right)$ the expansion lasts linearly in world time $a(t) \sim t$. Notice, that these expressions are obtained without taking into account the radiation energy density and pressure, nevertheless they give age of the Universe close enough to real one, since a radiation dominated epoch is more than two orders less than the present age of the Universe.

From the equation for a light ray one can find an angle $\chi_{0}$ :

$$
\cos \frac{\chi_{0}}{2}=1-\frac{t_{0}}{2 a_{m}}
$$

As we see, photons emitted at beginning of expansion at the moment of the maximum expansion $\left(t_{m}=2 a_{m}\right)$ have time to reach only the opposite pole $(\chi=\pi)$. As show the observational data [8] $b \sim 0.2$, so the observing relic radiation has been emitted from regions $\chi \sim 50^{\circ}$, i.e. closer than equator of the universe. Therefore, still direct effects of periodicity should not be observed.

In the $K_{m}$ frame 4-speed of a photon at $d \theta=d \varphi=0$ is: 


$$
u_{\mu}=\left(1, \dot{a},\left(1-\dot{a}^{2}\right)^{1 / 2}, 0,0\right) .
$$

i.e. the spatial components of 4 -speed are non-zero on the plane $(a, \chi)$, so speed of a photon's displacement on the circle's perimeter is less than the light speed and increases at the expansion:

$$
c_{(3)}(a)=\frac{a \delta \chi}{\delta t}=\sqrt{1-\dot{a}^{2}}<1 .
$$

This speed $c_{\chi}(a)$ increases at approaching the observer at later epoch and the photons from distant objects are accelerated as approaching us. This effect is similar to the restoring of the slowed down light speed at escaping by photons a region with a strong gravitational field.

\subsection{The relativistic redshift and parameters of the Universe}

Observable consequences of the closed model and parameters of the Universe have been considered in the previous paper [8] and for this reason here we present main results only. New effects are related by geometry fo 3-sphere, kinematics with relativistic effects and also by a combination of these two aspects.

New optical effects arise because of 3-sphere expansion in 4-space with radial velocity $\dot{a}$, orthogonal to the direction of radiation emission. It leads to the transverse Doppler-effect reducing frequencies with the factor $(1+z)^{1 / 3}$ and to the relativistic aberration reducing luminosity with the factor $(1+z)^{2 / 3}$.

The element of solid angle $d \Omega_{r}=d\left(\cos \theta^{\prime}\right) \cdot d \varphi$ under which the source is visible in the rest frame of receiver, will differ from the same solid angle $d \Omega_{e}=d(\cos \theta) \cdot d \varphi$ in the rest frame of the source and the apparent luminosity $l$ should be multiplied to the factor:

$$
\frac{d \Omega_{e}}{d \Omega_{r}}=\frac{1-\dot{a}_{z}^{2}}{1-\dot{a}_{0}^{2}}=\frac{a_{z}}{a_{0}}=\frac{1}{(1+z)^{2 / 3}} .
$$

Definition $l$, containing also the factor $(1+z)^{-2}$ from energy and frequency of arrival of photons, now takes a form:

$$
l=\frac{L}{4 \pi d_{h, 0}^{2}}=\frac{L}{4 \pi a_{0}^{2} \sin ^{2} \chi_{z}} \cdot \frac{1}{(1+z)^{8 / 3}},
$$

where $L$ is absolute luminosity, $d_{p, 0}$ is luminosity distance. This gives:

$$
\begin{gathered}
d_{p, 0}=a_{0} \sin \chi \cdot(1+z)^{4 / 3}=10^{-5+\mu / 5} M n c, \\
\mu=5 \lg \left[a_{0}(1+z)^{4 / 3} \sin \chi\right]+25 .
\end{gathered}
$$

From the equation for the light ray it follows:

$$
\chi_{z}=\int_{a_{z}}^{a_{0}} \frac{d a}{a} \cdot \frac{d \tau}{d a}=\int_{a_{z}}^{a_{0}} \frac{d a}{\sqrt{a\left(a_{m}-a\right)}}=\arcsin \left(1-\frac{2 a_{z}}{a_{m}}\right)-\arcsin \left(1-\frac{2 a_{0}}{a_{m}}\right) .
$$

Inserting (7) and (25) into (24), we obtain new "luminosity-redshift" relation:

$$
\mu=5 \lg \{3(1-b) Z[Z-1-(1-2 b)(\sqrt{(Z-b) /(1-b)}-1)]\}+A,
$$

where $Z \equiv(1+z)^{2 / 3}$ and 


$$
A=5 \lg \left(c / H_{o b s}\right)+25, \quad H_{o b s}=\frac{3}{2} H_{0}=\frac{3 c}{2 a_{0}} \frac{1}{\sqrt{b^{-1}-1}} .
$$

The formula (26) is in agreement very well with the data for redshifts of SN 1a [8] and replaces Mattig's formula of former models without relativistic redshift. At small $z$ Eq. (26) simplifies and turns to:

$$
\mu \simeq 5 \lg \left(z+\gamma z^{2}\right)+A, \quad \gamma(b)=(4-5 b) / 6(1-b) .
$$

Comparison of the model with observations in [8] ( $\mu-z$ diagram for SN 1a) has allowed to estimate two basic parameters: $b$ and $H_{o b s}$. At neglecting the stasis effects they are approximately equal to: $h_{0}=0.698 b=0.18 \pm 0.07$ and with a minimal accounting the stasis effects in galaxy clusters (at $f_{0}=0.15$ ):

$$
h_{0}=0.734, \quad b=0.15 \pm 0.05 \text {. }
$$

Further all estimations of parameters of the universe we will perform on the basis of (29).

Present and maximal radii of the universe follow from (27):

$$
a_{0}=\frac{3 c}{2 H_{o b s}} \sqrt{b^{-1}-1}, \quad a_{m}=\frac{a_{0}}{b}
$$

and at the choice (29) are equal to $a_{0} \simeq 13 \mathrm{Gpc}, a_{m} \sim 70 \mathrm{Gpc}$.

Proper time age of the dust matter stage follow from (17) and (29), and is equal to $\tau_{0} \simeq 13 \mathrm{Gyr}$ (in world time $t_{0} \simeq$ of $47 \mathrm{Gyr}$ ). The proper time age at the maximal expansion $\tau_{m} \simeq 370 \mathrm{Gyr}\left(t_{m} \simeq 500 \mathrm{Gyr}\right)$.

Total mass of the universe $M$ and total matter density $\rho_{0}$ are determined by the value $a_{m}(12)$ :

$$
\begin{gathered}
M=\frac{a_{m} c^{2}}{2 G}=1.5 \cdot 10^{57} \mathrm{~g} \simeq 1.5 \cdot 10^{13} \bar{M}_{\mathrm{gal}} . \\
\rho_{0}=\frac{M}{2 \pi^{2} a_{0}^{3}} \simeq 11,6 \cdot 10^{-31} \mathrm{~g} \cdot \mathrm{cm}^{-3}
\end{gathered}
$$

Dark matter density is defined as difference between $\rho_{0}$ and the observing baryon density $\rho_{b} \simeq 3.4 \cdot 10^{-31} \mathrm{~g} \cdot \mathrm{cm}^{-3}$ (30\% of $\rho_{0}$ ). At the chosen parameters it is predicted as equal to $\rho_{d m} \simeq 8.2 \cdot 10^{-31} \mathrm{~g} \cdot \mathrm{cm}^{-3}$ or $70 \%$ of $\rho_{0}$.

Changing with distance a number density of sources as $\sin \chi_{z}$ (w.r.t. a flat case) may be detected by number counting in the range of a 3 -angle $\chi, \chi+\Delta \chi$. Since the model gives new dependence of distance on the redshift, then there also takes place a new dependence of the number densities on the redshift.

Changing of apparent sizes and surface luminosities and some other standard geometrical effects of spherical geometry, also determined by $\sin \chi_{z}$ and new formulas for distances, are given in most of the literature. All of them are observable and also can be exhibited as specifically universal properties of distant objects.

Changing of length of path of photons in expanding 3-sphere with respect to a path in flat space leads to new corrections to intergalactic scattering and absorption. 
Consequences of placing of sources at larger radius $a_{z}$ change observable properties of sources at given $z$ with coefficient $(1+z)^{1 / 3}$ (in comparison with former models) also are numerous and should be taken into account at interpretation of results of observations.

Renormalization of the gravitational constant in the closed world is related also by a gravitational self-action of objects on "round-the-world" paths, similar to an elastic force in a closed chain. Therefore gravitational resistance to expansion of the closed universe of finite radius as a whole will be stronger than in a flat space. The result of such effective strengthening of the gravitational attraction can be taken into account as in particle physics and condensed media - by "renormalizing" the gravitational constant $G$ :

$$
G_{\text {eff }}=\alpha \cdot G, \quad \alpha>1 \text {. }
$$

In the right hand side of Einstein's equations such effective increasing of the gravitational constant at given density is equivalent to the increasing of the matter density at unchanged gravitational constant:

$$
G_{\text {eff }} \rho=(G \alpha) \rho=G(\alpha \rho)=G \rho_{\text {eff }} .
$$

Effective dark matter can be introduced at the second way of taking into account of last effect when the gravitational constant is left unchanged, but instead of the usual matter density $\rho_{m}$ a higher density $\rho_{m, e f f}$ can be introduced:

$$
\rho_{m, e f f}=\alpha \cdot \rho_{m}=\rho_{m}+\rho_{d},
$$

where $\rho_{d}$ is the density of "effective dark matter".

\section{Early Universe at nonzero pressure}

\subsection{Closed universe in 4 -space at nonzero pressure}

At early epochs with nonzero pressure the first of Einstein equations (92) remained the same as for the dust matter, but at the radiation dominated epoch, due to the second equation (93), the expansion law changes.

The Eq. (93) can be simplified by using Eq. (92). By rewriting it as:

$$
\frac{d}{d t}\left(\frac{1}{1-\dot{a}^{2}}\right)=-\frac{1}{3} \kappa a \dot{a}(\rho+3 p)
$$

and, using Eq. (92) again, we transform it to the form:

$$
\frac{d}{d t}\left(\rho a^{2}\right)=-a \dot{a}(\rho+3 p) \text {. }
$$

It gives the evolution equation for the energy density and pressure:

$$
\frac{d \rho}{\rho+p}=-3 \frac{d a}{a} \text {. }
$$

Then we take into account that the energy density is sum of two components - the matter density $\rho_{m}$ and the radiation density $\rho_{r}$ :

$$
\rho=\rho_{m}+\rho_{r}, \quad p_{r}=\rho_{r} / 3 .
$$

If in addition we use the fact that at all epochs the matter pressure is sufficiently smaller than the radiation pressure $p_{m} \ll p_{r}$ and it can be neglected, the model again simplifies and can be exactly solved. Considering the relationships: 


$$
\rho_{r} a^{4}=\text { const. } \quad \frac{\rho_{r 0}}{\rho_{m 0}}=w_{0} \sim 10^{-5},
$$

the sum of density and pressure in Eq. (38) can be represented in the form:

$$
\rho+p=\frac{1}{3} \rho_{m 0} a_{0}^{3}\left(\frac{3}{a^{3}}+\frac{4 w_{0} a_{0}}{a^{4}}\right) .
$$

As the result, Eq. (38) transforms to:

$$
d \rho=-\rho_{m 0} a_{0}^{3}\left(3 \frac{d a}{a^{4}}+4 w_{0} a_{0} \frac{d a}{a^{5}}\right),
$$

solution of which is

$$
\rho=\rho_{m 0} \frac{a_{0}^{3}}{a^{3}}\left(1+w_{0} \frac{a_{0}}{a}\right) .
$$

At large radius $a \sim w_{0} a_{0} \sim 10^{-5} a_{0}$ this gives the dependence for the dust matter $\rho \rightarrow \rho_{m} \sim a^{-3}$, and at small radii $a \ll w_{0} a_{0}$ leads to the dependence for radiation $\rho \rightarrow \rho_{r} \sim a^{-4}$.

Further from Eq. (92) we directly obtain the expressions for the time dilation factor:

$$
\sqrt{1-\dot{a}^{2}}=\frac{a}{a_{r m}} \frac{1}{\sqrt{1+a / w_{0} a_{0}}}
$$

where it is denoted $a_{r m}^{2}=w_{0} a_{0} a_{m}$, and for the expansion (contraction) speed:

$$
\dot{a}= \pm \sqrt{1-\frac{a}{a+w_{0} a_{0}} \cdot \frac{a}{a_{m}}} .
$$

Last expression gives the relation for the world time age at the expansion:

$$
\begin{aligned}
& t=\int \frac{d a}{\sqrt{1-a^{2} / a_{m}\left(a+w_{0} a_{0}\right)}} \approx \\
& \approx \int d a \sqrt{1+\frac{a^{2}}{a_{m}\left(a+a_{0} w_{0}\right)}} \approx a+\frac{1}{2 a_{m}} \int d a \frac{a^{2}}{a+a_{0} w_{0}} .
\end{aligned}
$$

Here in the integral, which cannot be taken directly, the denominator is expanded at early epochs by using $a+w_{0} a_{0} \approx a^{2} / a_{m}$, so for the world time age in this approximation we have:

$$
t=a\left(1-\frac{w_{0} a_{0}}{2 a_{m}}+\frac{a}{4 a_{m}}\right)-\frac{w_{0}^{2} a_{0}^{2}}{2 a_{m}} \ln \left(1+\frac{a}{w_{0} a_{0}}\right) .
$$

The proper time interval of expansion takes a form:

$$
\tau=\int d t \sqrt{1-\dot{a}^{2}}=\int d a \frac{a}{\sqrt{a\left(a_{m}-a\right)+a_{r m}^{2}}}+C^{\prime},
$$

Here the integral can be taken exactly and the result for the proper time age is:

$$
\tau=\frac{a_{m}}{2}\left(\arcsin \frac{1}{\sqrt{1+4 b w_{0}}}-\arcsin \frac{1-2 a / a_{m}}{\sqrt{1+4 b w_{0}}}\right)+a_{r m}-\sqrt{a\left(a_{m}-a\right)+a_{r m}^{2}} .
$$


In the approximation $4 b w_{0} \ll 1$ this formula simplifies:

$$
\tau \approx a_{m} \arcsin \sqrt{\frac{a}{a_{m}}}+a_{r m}-\sqrt{a\left(a_{m}-a\right)+a_{r m}^{2}} .
$$

The ratio of the proper time intervals then has the form:

$$
\frac{\Delta \tau_{0}}{\Delta \tau}=\frac{\Delta t_{0}}{\Delta t} \frac{\sqrt{1-\dot{a}_{0}^{2}}}{\sqrt{1-\dot{a}^{2}}}=\frac{a_{0}^{2}}{a^{2}} \frac{\sqrt{w_{0}+a / a_{0}}}{\sqrt{1+w_{0}}} \approx \frac{a_{0}^{2}}{a^{2}} \sqrt{w_{0}+\frac{a}{a_{0}}}=1+z,
$$

which gives the formula for redshift in a general case:

$$
\frac{a_{0}^{2}}{a^{2}} \sqrt{w_{0}+\frac{a}{a_{0}}}=1+z
$$

Thus, at the simultaneous accounting of the energy densities of matter and radiation the basic relationships are complicate slightly and the model remains as exactly solvable. In fact formulas in general case are necessary only in a small interval of evolution time of the universe, when these two densities are the same order.

\subsection{The radiation dominated epoch}

At transition to the radiation dominated epoch the equations of the model once again simplifies so that it is easier to derive all relations from the beginning. At early epochs with $a \ll w_{0} a_{0}$ the equations (11) and (38), following from Einstein's equations (92)-(93), transform to the equations:

$$
\begin{aligned}
& \frac{1}{1-\dot{a}^{2}}=\frac{a_{r m}^{2}}{a^{2}}, \\
& \frac{d \rho_{r}}{\rho_{r}}=-4 \frac{d a}{a} .
\end{aligned}
$$

The solution of Eq. (54) is (40) and for the relativistic plasma of temperature $T$ we have the relationships:

$$
\rho=\rho_{r 0} \frac{a_{r 0}^{4}}{a^{4}}=\sigma T^{4}, \quad T=\frac{a_{r 0}}{a} T_{r 0} .
$$

The equation (53) directly gives the expressions for a time delay factor and an expansion speed:

$$
\sqrt{1-\dot{a}^{2}}=\frac{a}{a_{r m}}, \quad \dot{a}= \pm \sqrt{1-\frac{a^{2}}{a_{r m}^{2}}} .
$$

The Eq.(56) in the integral form:

$$
\int \frac{d a}{\sqrt{1-a^{2} / a_{r m}^{2}}}=t+C,
$$

has a simple solution (with zero initial value):

$$
a(t)=a_{r m} \sin \left(t / a_{r m}\right) .
$$

The proper time age in the radiation dominated epoch in comoving frame is:

$$
\tau=\int_{0}^{t} d t \sqrt{1-\dot{a}^{2}}=\int_{0}^{a} \frac{d a}{\sqrt{1-a^{2} / a_{r m}^{2}}} \frac{a}{a_{r m}}=a_{r m}\left(1-\sqrt{1-a^{2} / a_{r m}^{2}}\right),
$$

Thus, the dependences between $\tau, t$ and $a$, according to (58), have the form: 


$$
\begin{gathered}
\tau=2 a_{r m} \sin ^{2}\left(t / 2 a_{r m}\right), \\
a=a_{m} \sqrt{1-\left(1-\tau / a_{r m}\right)^{2}}=\sqrt{\tau\left(2 a_{r m}-\tau\right)} .
\end{gathered}
$$
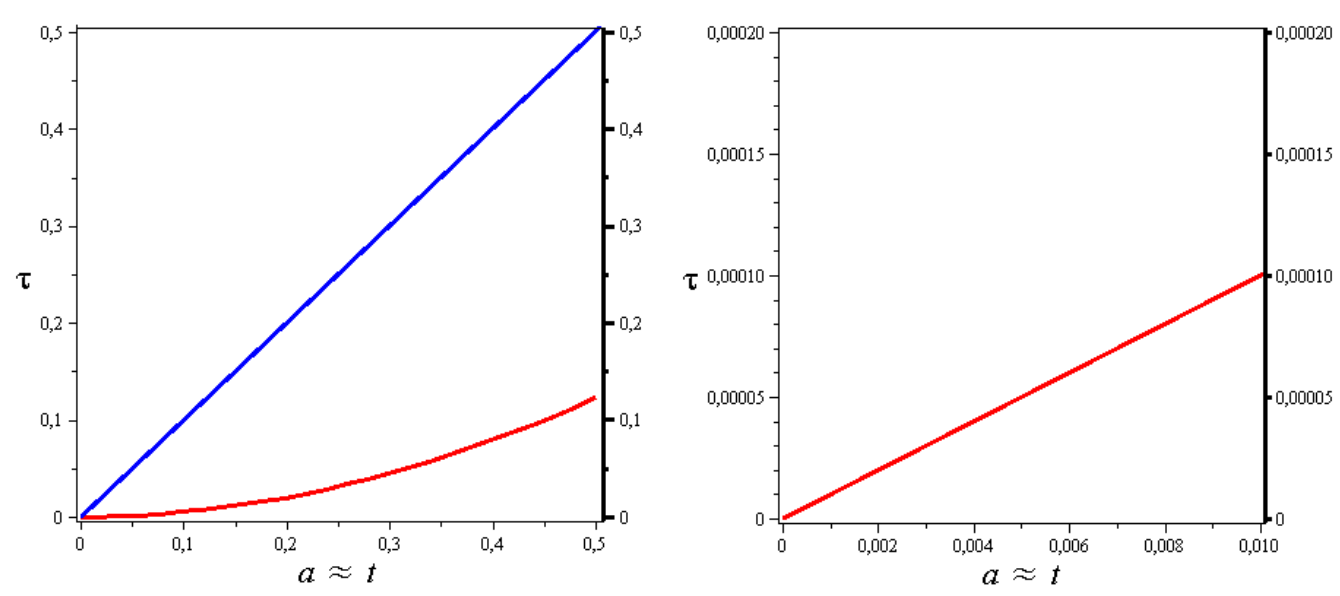

Fig. 1. The dependences $\tau(a)$ (red curve) and $t(a)$ (blue curve) at early epochs.

At very early epochs we obtain the approximated formulas:

$$
\tau \approx t \cdot \frac{t}{2 a_{r m}} \approx a \cdot \frac{a}{2 a_{r m}}
$$

and we also see that in this approximation a dependence of $\tau$ on $a$ is the same, as in Friedmann's model where $\tau=a^{2} / 2 a_{r m}$.

The presence of the time delay factor $a / 2 a_{r m}$ leads to the essential retardation of proper time moments from world time moments. As we can see from Fig. 1, this retardation can exceed 100 and more times and, as the result, the expansion becomes similar to an inflation when in terms of world time the local rate of physical processes is essentially slowed down, while the expansion occurs regularly and practically with the light velocity.

A ratio of proper time intervals is equal to

$$
\frac{\Delta \tau_{r}}{\Delta \tau}=\frac{\Delta t_{r}}{\Delta t} \frac{\sqrt{1-\dot{a}_{r}^{2}}}{\sqrt{1-\dot{a}^{2}}}=\frac{a_{r}^{2}}{a^{2}}=1+z_{r},
$$

and consequently:

$$
\frac{a_{r}}{a}=\sqrt{1+z_{r}} .
$$

Thus, at the early epochs the universe expands practically with the speed of light, the expansion law is simple and is given by Eqs. (58) and (62), the redshifts decrease at increasing of $a$ as in Eq.(64). This means that all processes occurring at certain temperatures, will occur at sufficiently larger values of the radius, i.e. at later periods than in the Friedmann model. This consequence of the model naturally leads to the expansion scenario similar to the inflationary. The physical reason for this is extremely simple and it aroused due to the strong relativistic delay of proper times at this period. Really, the radial expansion (increasing of 3-sphere's radius) goes almost with the light speed and consequently the azimuthal components of the light speed (along the 3-sphere) are very small and thus all processes on the 3-sphere also are highly slowed down. 


\subsection{The nucleosynthesis epoch}

The first describable epoch, the consequences of which are not only observable, but also can be described and explained within the known physics, is the nucleosynthesis epoch. In the present model the physical conditions at temperatures, when there were required concentration of free neutrons, and duration of that period, sufficiently differ from the conditions of former models. The concentrations are smaller, but instead the times become accordingly longer. As the result, the nucleosynthesis theory remains unchanged in specifically nuclear physics aspects, but the processes occur at sufficiently different cosmological conditions. Further we will consider only the general estimations of an order of quantities only.

A period between annihilation of electron-positron pairs at $1 \mathrm{MeV}$ or $1.2 \cdot 10^{10} \mathrm{~K}$ and freezing of neutrons at temperature of $0.7 \mathrm{MeV}$ or $8.1 \cdot 10^{9} \mathrm{~K}$ in the present model corresponds to the proper age interval of the universe $\tau \approx 1.22 \div 1.81 \mathrm{yr}$ (world time age $t \approx 9.3 \div 12 \mathrm{yr}$ ), and the radii $a \approx 9.3 \div 12 \mathrm{ly}$.

The concentration of nucleons thus was larger in $n / n_{0}=(1.0 \div 0.5) \cdot 10^{20}$ times or, at the present value $n_{0} \approx 2 \cdot 10^{-7} \mathrm{sm}^{-3}$, it was of order $n \approx(2.0 \div 1.0) \cdot 10^{13} \mathrm{sm}^{-3}$. The radiation energy density was in $10^{26}$ times larger than the present day density. At this period, the duration time of which on proper time is $0.59 \mathrm{yr}$ or $\Delta \tau \approx 1.8 \cdot 10^{8} \mathrm{sec}$, scatterings of nucleons with energetic photons and neutrino were intensive and they were enough for slowly decreasing the number of free neutrons.

At former calculations of nucleosynthesis in the framework of Friedmann models there were made an assumption that during the period after freezing of neutrons the concentration of nucleons was of order $n / n_{0} \approx 10^{25}$, i.e. $n_{p} \approx 10^{18} \mathrm{sm}^{-3}$, and the duration time of nucleosynthesis was an order of decay time of neutron $\Delta \tau \approx 10^{3} \mathrm{sec}$. An estimation of number of scatterings of nucleons $Q$ during $\Delta \tau$ at the cross-section $\sigma \sim 3 \cdot 10^{-26} \mathrm{sm}^{2} / \mathrm{sec}$ and velocity $\mathrm{v} \simeq 3 \cdot 10^{8} \mathrm{sm} \cdot \mathrm{sec}^{-1}$ has been performed by the formula:

$$
Q \simeq \sigma \mathrm{v} \cdot n_{p} \Delta \tau \text {. }
$$

Under these conditions in the Friedmann model $n_{p} \Delta \tau \sim 10^{21} \mathrm{sm}^{-3} \mathrm{sec}^{-1}$ and for the number of scattering it has been obtained $Q_{F r} \approx 10^{3} \div 10^{4}$.

In the present version of the closed model the cross sections and the velocities are the same, but the concentrations of nucleons is less in $10^{5}$ times, the energy densities of radiation and neutrino $10^{6}$ times lower than in the former models. From the other side, the duration time, when there were free neutrons, was in $10^{5}$ times longer. The radiation and neutrino densities were enough for slow decreasing of the number of neutrons, while the number of collisions of neutrons by protons and then by formed deuterium remained approximately as in former approaches.

As a result in the present model $n_{p} \Delta \tau \sim 10^{21} \mathrm{sm}^{-3} / \mathrm{sec}$ and the formula (65) gives $Q_{\text {new }} \approx 10^{3} \div 10^{4}$. It is enough for the main part of neutrons to be captured into deuterium, and further deuterium had time to capture a free neutron, by passing into $\mathrm{He}^{3}$. The synthesis goes also in the form of merging of deuterium.

In the former models the nucleosynthesis epoch lasted of order the decay time of neutron. As the result, the observing high abundance of deuterium was a problem since 
all deuterium, not passed into helium, should be destroyed at required high concentrations of nucleons. This then had led to far-reaching conclusions that the closed model is inadmissible due to such densities, etc.

In the present model the nucleosynthesis is related by slow decreasing of concentration of free neutrons at increasing of the concentrations of deuterium and helium during about half year from the period of annihilation of $e^{+} e^{-}$pairs up to the reaching of the freezing temperature of neutrons and slightly later. The small concentration of nucleons is the important advantage of the new model for a natural explanation of the present high abundance of deuterium (of order $10^{-5}$ ). Since the synthesis of deuterium D and following synthesis of $\mathrm{He}^{3}, \mathrm{He}^{4}$ and other light elements in the present model goes longer, then, due to the smallness of concentrations in $10^{6}$ times, the disintegration of light nucleus, in particular, deuterium and $\mathrm{He}^{3}$, lasted sufficiently slower, which thus accumulated.

The relation of equilibrium concentration of neutrons and protons at this period is defined basically by the freezing temperature of neutrons and practically does not depend on details of cosmological models. Here we present the standard derivation to show simplicity of a physical picture. At $T_{n}=0.7 \mathrm{MeV}$, with taking into account $\Delta m=m_{n}-m_{p}=1.3 \mathrm{MeV}$, the relation of equilibrium concentrations is equal to:

$$
n_{n} / n_{p}=\exp \left(-\Delta m / T_{n}\right) \simeq 1 / 6.4
$$

As the result, during the nucleosynthesis, to each pair of neutrons forming further $\mathrm{He}^{4}$ nucleus, there were averagely $2 \times 6.4 \approx 13$ protons, two of which will be captured by forming $\mathrm{He}^{4}$. Therefore, to a one nucleus of $\mathrm{He}^{4}$ there remained about 11 free protons. Then we come to the standard estimation for the relative abundance of hydrogen and helium in terms of concentrations and masses:

$$
\begin{aligned}
& \frac{n_{H e^{4}}}{n_{H^{1}}+n_{H e^{4}}} \simeq \frac{1}{12} \simeq 8 \%, \\
& \frac{M_{H e^{4}}}{M_{H^{1}}+M_{H e^{4}}} \simeq \frac{4 m_{p}}{11 m_{p}+4 m_{p}} \simeq \frac{4}{15} \simeq 26 \% .
\end{aligned}
$$

For the estimations the agreement with observational data $8 \%$ and $24 \%$ is good enough.

As it is known, though some aspects of nucleosynthesis are sensitive to kinetics of various processes, nevertheless the final relations of the concentrations are quite universal since mainly depend on the temperatures and nuclear physics aspects. Therefore, as it has been demonstrated by the above presented estimations, the synthesis of light elements even at the presented sufficiently different concentrations and times, allows us to explain their present abundance. Results of more detailed calculations of nucleosynthesis in the model will be presented in forthcoming publications.

\section{Early Universe at zero pressure}

\subsection{Expansion and contraction of a thin dust 3-sphere in 4-space}

In the closed model of the universe as expanding 3-sphere in 4-space, at a dust matter domination epoch the evolution equations in the rest frame of the 3-sphere takes the extremely simple form (14). As we see, firstly, during the early periods $t \ll a_{m}$ the radius grows on world time almost linearly: $a \simeq t$, and, secondly, at given value of $z$ 
the radius $a_{z} \sim(1+z)^{-2 / 3}$ in $(1+z)^{1 / 3}$ times larger than in former models where the relationship was $a_{z} \sim(1+z)^{-1}$.

The proper time age (17) at early periods takes the form:

$$
\tau \simeq a \cdot \frac{2}{3} \sqrt{\frac{a}{a_{m}}}\left(1+\frac{3 a}{10 a_{m}}\right)+O\left(a^{3}\right) .
$$
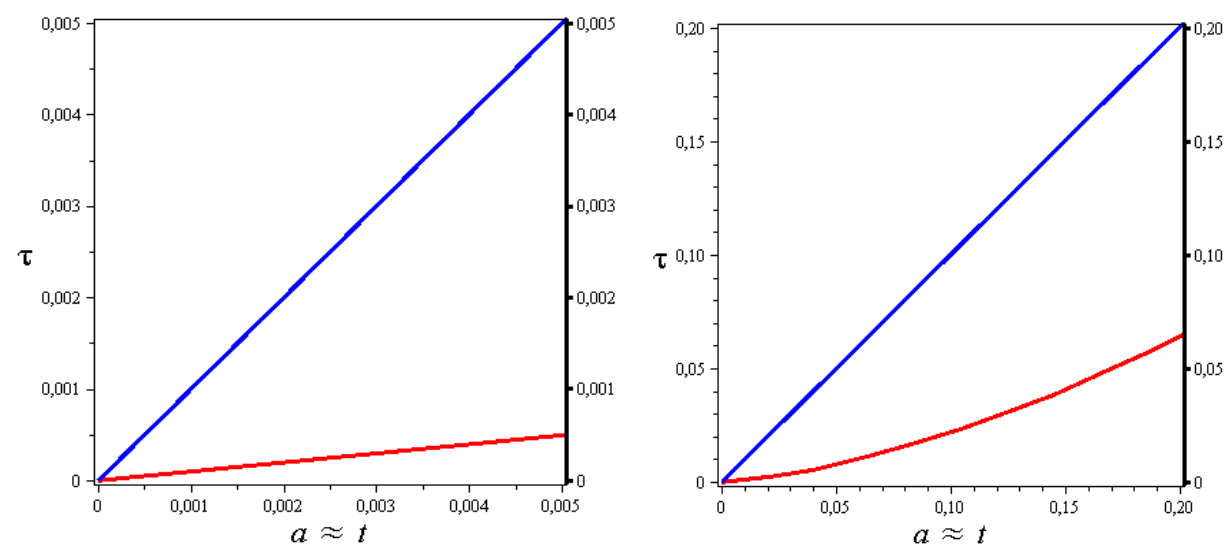

Fig. 2. The dependences $\tau(a)$ (red curve) and $t(a)$ (blue curve) from (68).

Thus, because of the presence of the factors containing a small parameter $a / a_{m}$, the proper time goes many times slowly than world time. As it is clear from Fig.2, the time dilation factor exceeds $4 \div 10$ times and more.

\subsection{The recombination epoch}

In the present model, to photons arriving from the recombination epoch with redshift $z$ corresponds a scale factor according Eq. (52). As the result, to the local temperatures $\quad T \approx\left(1100^{\circ} \div 4000^{\circ}\right) \mathrm{K}$ correspond redshifts $z \sim(3,3 \div 0.9) \cdot 10^{3}$ ("unperturbed" by the stasis effects [13]), the radii $a \sim 193 \div 456$ Mly and the proper age $\tau \sim 3.7 \div 13.3 \mathrm{Myr}(t \sim 193 \div 456 \mathrm{yr})$.

The duration time of the recombination epoch is $\Delta \tau \sim 9.7 \mathrm{Myr}(\Delta t \sim 263 \mathrm{Myr})$ and at this epoch the universe's radius increased in 2.4 times. As the result, the average density of matter was only in $10^{7} \div 10^{6}$ times higher $\rho_{0}$ and it was $\rho_{\text {rec }} \sim\left(10^{-23} \div 10^{-24}\right) \mathrm{g} \cdot \mathrm{cm}^{-3}$, which is of order the present average matter density in large galaxies. Previous calculations of the characteristics of CMB have been based on estimations of density $\rho_{\text {rec }}$ in $10^{3} \div 10^{4}$ times exceeding than in the present model.

The distance passed by a photon through the inhomogeneities is proportional to a mean time between collisions $\tau_{c}$ multiplied to the number of collisions $N \approx \sigma_{T} n_{e} / \tau_{c}$, where $n_{e}$ is the concentration of electrons and $\sigma_{T}$ is the Thomson scattering crosssection. By dividing this density to the baryon mass we find a concentration of nucleons and thus electrons: $n_{e} \sim\left(10^{4} \div 10^{2}\right) \mathrm{cm}^{-3}$. Since $\sigma_{T} \simeq 0.7 \cdot 10^{-24} \mathrm{~cm}^{-2}$, we find the estimation:

$$
\tau_{c} \sim\left(c n_{e} \sigma_{T}\right)^{-1} \simeq(0,5 \div 5) \times 10^{11} \mathrm{sec}
$$


The duration time of the recombination $\Delta \tau_{\text {rec }} \approx 3 \times 10^{14}$ sec exceeds the mean scattering time $\Delta \tau_{c}(69)$ to 3 orders, i.e. during the recombination occur of order $10^{3}$ scatterings of photon with electrons.

The concentration of particles at this epoch was so small that we may neglect by mutual scattering of particles, the pressure of matter and sound in fact there practically absent. There really occur, besides the gravitational contraction of clouds, the interactions of particles by the photons only before loss of direct contact between the relic flow and matter. Thus, further it is enough to consider only a fading diffusion of electrons, ions and neutral atoms in a sufficiently denser photon gas.

The stasis of temperature and photon density of CMB flow at crossing the gravitationally-bound regions (GBR), such as galaxy clusters, has been studies in the paper [13]. It has been shown that due to switching out from the cosmological expansion a part of the relic flow, intercrossing clusters remain warmer w.r.t. normally expanded flow beyond the clusters.

As a result, instead of the former paradigm about almost sterile propagation of the relic flow since the recombination epoch, in fact, from GR and the cosmological principle follows an opposite picture. Mixing of flows of relic photons many times isolated in GRBs from the expansion flow, essentially changes the physical characteristics of CMB, particularly, leads to its isotropy and loss of information about perturbations at early epochs.

The observing angular anisotropy follows from the stasis effects at crossing of CMB flow in multiple layers of nearby galaxy clusters at latest time.

\section{The life cycle of the Universe and cosmological problems}

\subsection{The life cycle of the Universe}

\section{A) Big Bang and very early Universe}

In the simple model of the closed universe in the real 4-space up to now the effects of gravitation along 4-th dimension were not considered and all effect from this dimension of space has been reduced to kinematic effects. However at earliest stages the radius becomes nearer to a gravitational radius along 4-th dimension which inevitable appears in solutions of Einstein's equations. As the result there is necessary to consider the gravitational delay of proper times and the contraction of radial distances (see [11]).

The solutions of Einstein's equations for 3-sphere filled by dust matter are wellknown (references see in [11]) and for the proper time delay give the expression:

$$
d \tau(t)=d t \sqrt{1-\frac{a_{g}^{2}}{a^{2}}} \sqrt{1-\frac{\dot{a}^{2}}{1-a_{g}^{2} / a^{2}}}, \quad a_{g}^{2}=a_{m} a_{l},
$$

where $a_{m}=2 G M$ is the maximal radius of expansion and $a_{l} \approx \delta a \geq l_{p l}$ is a certain length characterizing the "thickness" along the radial direction of 3 -sphere which cannot be less than the Planck length $l_{p l}$.

Numerically $a_{m} \approx 10^{28} \mathrm{~cm}$ is so large that a value of $a_{l}$ correspondingly should be so small. Since a simple kinematical model well enough describes the data, then $a_{l}$ is small and it is natural to take for it a most "pessimistic" value and still to identify it with the Planck length: 


$$
\begin{aligned}
& a_{l} \approx l_{p l} \approx 10^{-33} \mathrm{cM}, \\
& a_{g}=\sqrt{a_{m} \cdot l_{p l}} \approx 10^{-2} \mathrm{cM} .
\end{aligned}
$$

In that case the Planck density, when there occurs a gravitational freezing of neighbor particles, should be reached at $a \rightarrow a_{m} \approx 10^{-2} \mathrm{~cm}$. If we neglect the gravitational time delay, the energy density will be of order of the Planck density $\rho_{p l} \approx \rho_{0} \cdot 10^{125} \approx 10^{95}$ of $\mathrm{g} \mathrm{cm}^{-3}$ at $a \approx 10^{-2} \mathrm{~cm}$ and $t \approx 10^{-15} \mathrm{sec}$.

At these densities it is necessary to consider hierarchy of structures of particles on the basis of the Standard Model, and possible models of unification or substructures of known particles. Here we approach the borders of known physics where the cosmological theory passes into the area of the cosmological hypotheses.

The Big Bang mechanisms also concern to the questions, the solutions of which are unclear in the known physics. But until all opportunities of modeling by using known particles, fields and probable physical conditions at that epoch should be settled, these problems also should be carried out to problems related only with complexity of structures and interactions, but not with uncertainty.

\section{B) Evolution of the universe in the expansion era}

At the present value of radius $a_{0} \approx 13 \mathrm{Gpc}$ and maximal radius of expansion $a_{m} \approx 70 \mathrm{Gpc}$ the expansion era will last on the proper time about $370 \mathrm{Gyr}$, from which still passed $4 \%$ only.

This era, from the Big Bang and up to the present time, includes the epochs from which there are observable evidences:

1. Early epoch when a particle content has been formed;

2. Nucleosynthesis epoch when light nuclei were formed;

3. Recombination epoch when light atoms were formed;

4. Structure formation epoch when galaxies and stars were formed;

5. Modern epoch.

In the present model the scenarios of the first three epochs require sufficient updating with taking into account the new physical conditions - lower densities or concentrations, and also longer duration times. The further development of inhomogeneities into stars and galaxies (4-th and 5-th periods) practically unrelated with specifically relativistic effects, but the present model enters many modifications into the cosmological picture in these periods also.

The distances up to high redshift sources will essentially change, especially, up to quasars, GRB and galaxies, essentially increase a number of sources and the mass of the universe because of increased volume at the given radius. Now the redshifts will connect with distances differently, as well as the apparent luminosities with the absolute luminosities. The estimations of the distances between the sources, the times of epochs when the signals have been emitted and the estimations of absorption and scattering in the intergalactic environment will change also. The characteristics of high $z$ QSOs and GRBs will change also because of the new distances up to them. All these modifications have observable consequences and at the further confrontation of the model with observations the scenarios of evolution of objects will be specified and the parameters of the universe will be more precisely determined.

During the subsequent evolution of the universe up to stopping of the expansion tens generations of medial mass stars will have time to flash and decline. At end of this era the formed compact objects (white dwarfs, hadronic stars and frozars) will represent 
main stellar population of galaxies. Galaxies after numerous merging get to be consolidated so that the central parts of many of them can turn again to a hot stage of evolution analogous to quasars. Then an observable picture of such hypergalaxies will be essentially differing from the present one and their central parts will be much brighter than their rim.

The distances between the gravitationally bound galaxy clusters will be increased several times whereas the sizes of many of them will essentially decrease. In addition to the compact clusters, which become clearly exhibited, there will be formed the superclusters too.

The CMB temperature at the end of the era will be two orders lower and the distortions from clusters and superclusters become insufficient for the angular distribution of the relic radiation temperature.

\section{C) The contraction era and its peculiarities}

The duration time of the contraction era is also about $370 \mathrm{Gyr}$, and together with remained time of the expansion era, before the full contraction and the Big Collapse remains of order $700 \mathrm{Gyr}$.

The contraction era will differ first of all by the closing in of clusters of galaxies and other sources and violetshift of their spectral lines (there remain zero shift and redshift for distant sources also). Other difference will be related by hypergalaxies, having bright nuclei and a great number of compact objects, in particular, frozars [11], will dominate.

In the process of contraction and achievement of temperatures of ionization of hydrogen, helium and other elements, most part of nonluminous matter also will pass to the plasma state.

However, since the matter will be concentrated mainly in the compact objects, particularly, at merging of white dwarfs and neutron stars, there will be formed at first single frozars, and then there will be formed the frozar clusters from three, four and multiple frozar complexes members of which will froze at fixed distances. Notice that three frozars cannot already merge, since the gravitational radius of the system approximately three times exceeds the gravitational radius of each of them and they will freeze on some distance from the center of mass of the system. Similar process with nucleus of galaxies and quasars will lead to the formation of the frozen complexes from supermassive frozars.

Thus, the contraction era will essentially differ from the expansion era since the most part of matter will be frozen in complexes with more and more growing gravitational radii. Finally these complexes will prevent also to the further contraction of the universe as a whole and the contraction to a very small volume will not occur.

\section{D) The Big Collapse as the Big Freezing}

At formation of complexes of compact frozen objects and their merge finally into a total super complex in scales of the universe, the Big Collapse stops with transition into the Big Freezing.

However, if before of this freezing some processes lead to stopping of contraction and to the subsequent expansion, the new cycle will begin. But, this cycle will not be similar any more on the previous one since most part of the matter remains as frozen in the form of the frozar complexes which do not depend in any way on the cosmological expansion and this frozen matter will appear as a dark matter. 
In the present paper the problems of the possible cyclic evolution will not be considered since for this purpose while there are no bases. Further only some aspects of this problem will be considered in connection with the main cosmological problems.

\subsection{The lack of former main cosmological problems}

A) The lack of the flatness and the fine-tuning problems

In the closed model in 3-space at the early periods the space becomes flat with high accuracy. Really, the Friedmann equation can be written in the form:

$$
\Omega^{-1}-1=\frac{\text { const }}{\rho a^{2}}, \quad \Omega=\frac{\rho}{\rho_{c}},
$$

we see that for the ordinary matter and for the radiation dominating epochs there take place following relationships:

$$
\begin{aligned}
& \frac{\Omega_{0}^{-1}-1}{\Omega^{-1}-1}=\frac{\rho a^{3}}{\rho_{0} a_{0}^{3}} \frac{a_{0}}{a}=\frac{a_{0}}{a}, \\
& \frac{\Omega_{0}^{-1}-1}{\Omega^{-1}-1}=\frac{\rho a^{4}}{\rho_{0} a_{0}^{4}} \frac{a_{0}^{2}}{a^{2}}=\frac{a_{0}^{2}}{a^{2}}
\end{aligned}
$$

and, consequently, in both cases $\Omega \rightarrow 1$ at $a \rightarrow 0$.

In the closed model in 4-space the flatness problem is absent since in this model the Einstein equations lead to new evolution equation (53). At early periods it can be represented in the form, analogous to (72):

$$
\frac{1}{\Omega\left(1-\dot{a}^{2}\right)}-1=\frac{\text { const }}{\rho a^{2}},
$$

and, by using one again that $1-\dot{a}^{2}=a^{2} / a_{r m}^{2}$, we obtain:

$$
\frac{\left(a_{r m}^{2} / \Omega_{0} a_{0}^{2}\right)-1}{\left(a_{r m}^{2} / \Omega a^{2}\right)-1}=\frac{\rho a^{4}}{\rho_{0} a_{0}^{4}} \frac{a_{0}^{2}}{a^{2}}=\frac{a_{0}^{2}}{a^{2}} .
$$

As we see, at decreasing of radius $\Omega$ and the curvature increase monotonically, which is natural:

$$
\Omega \rightarrow \frac{a_{r m}^{2}}{a^{2}}, \quad a \rightarrow 0 .
$$

Thus, the model is free on the flatness problem and we not need in fine tuning.

\section{B) Homogeneity and isotropy as initial conditions}

Similar to models of spherical stars, a large-scale homogeneity and isotropy of the matter distribution in the closed cosmological model are the starting conditions providing the spherical symmetry and should not be consequences of a causal connection of different regions. If the initial distribution was averagely homogeneous and isotropic, it will remain such during expansion.

During several mln. years of radiation dominated epoch and also almost $10 \mathrm{Myr}$ of the recombination epoch the matter's particles diffused as a small admixture in a hightemperature gas of photons and this diffusion smoothed any sufficient inhomogeneity in the matter's distribution in the causally connected regions.

The front of relic radiation observed in our epoch has passed more than 13 Gly and has been perturbed continuously by the redshift and intensity stasis effects in 
gravitationally-bound inhomogeneities, such as galaxy clusters. Any small region of this front have been formed by mixing of radiation from large number of surrounding regions.

\section{C) The lack of the horizon problem}

The horizon problem in the Friedmann models has appeared due to the increasing the size of a causally-connected region (horizon) by the light speed $r_{h o r} \approx t$, while the scale factor raises more slowly as $a \approx t^{1 / 2}$ in early epochs and as $a \approx t^{2 / 3}$ later. On the other hand, the relic radiation has ceased to interact with matter after the recombination epoch and radiation densities in causally non-connected areas could not be correlated in any way, while in practice from them the averagely isotropic and uniform flows arrive to us.

In the present model of 3-sphere in 4-space the situation is opposite - the radius of the universe grows during all epochs more promptly, than a size of the causallyconnected region, especially in early periods. At the early periods the increasing of the universe's radius occurred almost with the light velocity $a \approx t$ in terms of the world time, while the size of the causally-connected region rose as $r_{h o r} \approx \tau$ in terms of the proper time which at early epochs was sufficiently slower than world time $\tau \ll t$. In this situation the initial spherical symmetric distribution of radiation dominate matter, required by the cosmological principle, will be conserved with high accuracy.

On the other hand, because of the stasis effects for radiation crossing of galaxy clusters former naive paradigm about "sterile" propagation of the relic flow should be rejected and replaced by new mechanism of mixing of differently perturbed segments of the relic flow. As a result, homogeneity of the observing CMB flow appears a consequence of large-scale homogeneity in matter distribution during the matter domination epoch.

Thus, in the present model the horizon problem is absent.

\section{D) The lack of the initial singularity problem}

As the closed universe in 4-space approaches close to a gravitational radius of the 3-sphere $a_{g}$ the proper times and the contraction process freezes in terms of the cosmological world time $t$ and the radius remains outside $a(t)>a_{g}$ at any finite moment $t<\infty$ [11].

This specifically relativistic effect is the same as for contraction of a thin dust 2sphere in 3-space, which freezes outside its gravitational radius $r_{g}=2 G M$ in the rest frame of the sphere, never reaching this radius $r(t)>r_{g}$ at any finite moment $t<\infty$. In this frame of reference between $r$ and $r_{g}$ at each finite moment of world time $t<\infty$ there is a small coordinate distance $\delta r(t)=r(t)-r_{g}$ to which there corresponds a spacelike proper distance interval:

$$
\delta s^{2}=-\frac{\delta r^{2}}{1-r_{g} / r}<0 .
$$

In GR this instantaneous interval of the proper radial distance in a rest frame is invariant just as the proper time interval in a spatial point of the same local frame. Thus, the spacelike character of the interval (78) is invariant and it remains as spacelike in any local frame at any physically admissible transformations of coordinates at $t<\infty$. 
For the closed universe as 3-sphere the situation mainly just the same, since here we have again

$$
\delta s^{2}=-\frac{\delta a^{2}}{1-a_{g}^{2} / a^{2}}<0 .
$$

Therefore at consecutive applying GR to the closed model the problem of the initial cosmological singularity does not appear.

Usually it has been believed that the problem arises when the process is described from the point of view of a comoving observer in terms of its proper time. But in this case GR also allows one to remove this problem.

In the former approaches it has been proposed that the contraction of the universe continues up to the Planck volume $V_{p l}$. However, each "point" particle is "an external observer" for another "point" particle and at contraction moves to any particle. As the result, at distances close $l_{p l}$ the proper times of each such particles becomes slowed down with respect to a common for the particles world time of the center of inertia of this pair.

Therefore, in fact, in GR it is necessary to consider contraction of each of $N_{0}$ present particles and formed $\Delta N$ quanta to volume $V_{p l}$, after which their wavelength will not decrease. As the result extremely small volume of the universe $V_{\min }$, up to which the contraction can be continued, is the sum of these elementary volumes $V_{p l}$ of particles and in terms of the Planck volume it always will be more than the present number of particles in the universe:

$$
V_{\text {min }}>N_{0} V_{p l} \sim 10^{88} V_{p l} .
$$

At reaching of this smallest volume $V_{\min }$ the particles become frozen due to proper gravitational fields and the cosmological contraction stops in terms of the cosmological proper time $\tau$ too. This restriction has a fundamental and model-independent character since follows from the classical (non-quantizing) component of the gravitational field.

\section{E) The lack of the entropy problem}

The problem of very large entropy arose due to that it has been supposed the contraction of the universe up to the elementary volume $V_{p l}$.

In the closed universe in 4-space the contraction continues only up to the smallest volume $V_{\min }$ from (80), where the particles become frozen in terms of the proper time and there no the problem of the large entropy. In the present model the total energy of the universe is non-zero and conserves just as the total energy of thin dust 2-sphere in 3space. A great number of elementary volumes $>10^{88}$ at maximal contraction and corresponding "number of cells" characterizing the large entropy of the universe also conserves, as the residual mean number of particles at the later periods of expansion.

\section{F) The lack of the cosmological constant}

The widely accepted former paradigm of cosmology mainly had based on the introduction of the cosmological constant $\Lambda$. However, its value has been found so small, that it cannot be explained not only by the Standard Model of particle physics, but by its hypothetical generalizations too. This is the cosmological constant problem, remained almost unsolvable in the former paradigms of cosmology and particle physics. 
In the previous papers on the vacuum energy I have argued that particle physics does not give neither theoretical, nor the observational bases for the non-zero vacuum energy. This means that cosmology can be in agreement with particle physics only at the lack of the cosmological constant $\Lambda=0$.

In the closed model in 4-space the cosmological constant is absent since the model is based on the standard Einstein's equations with the matter energy density only. This means that the model provides the long time wanted full agreement between cosmology and particle physics in the question of vacuum energy.

$\mathrm{G})$ The lack of the dark matter problem

Cosmological models determine limits for the dark matter density. From predicted by the closed model the total energy density $\rho_{0}$ we subtract the observing baryon density $\rho_{0 b} \sim 0.3 \rho_{0}$ and obtain the estimation for the dark matter density $\rho_{d m} \simeq 0.7 \rho_{0} \sim 8$, which reduced the problem to specifically astrophysical aspects.

To the cosmological astrophysical aspects concerns two new facts. Firstly, 3sphere's volume in $3 \pi / 2 \approx 4.71$ times exceeds the volume of a ball of the same radius in flat 3-space and, consequently, at the same local density the 3-sphere contains in 4,71 times more total mass than the ball.

Secondly, in the closed world there are two geodesics - the shortest and roundthe-world. The last one leads to additional gravitational interaction between sources which renormalizes the gravitational constant and additionally slower the expansion, especially at early epochs. This change of the gravitational constant also can be treated as presence in the universe of large fraction of an effective dark matter.

\section{$\mathrm{H})$ The charge asymmetry as an initial condition}

The lack of antiparticles (positrons, antiprotons and antineutrons) in the observable universe formally follows from the fact of $C P$-symmetry breaking in a hot phase. It leads to the excess of number of particles over antiparticles of order $10^{-8} \div 10^{-9}$ which is enough for the formation of the present baryons to photons ratio.

If in other approaches mainly the mechanisms of baryon charge non-conservation in the grand unification models have been used, in the present treatment more preferable seems the models of substructures, where the charge symmetry can be restored at level of substructures.

In other side, the $C P$-symmetry breaking can lead in the hot phase both to the excess of particles or antiparticles. The interchanging of the worlds and antiworlds in several cycles of the closed model the charge symmetry can be restored in such extended cosmological time scales of our universe.

And, finally, in the closed model the existence of the neighbor universes in 4space generally is possible, which can lead to an observable tidal action, distorting the structure of our universe. In this case in one of them at the hot phase it could survive the matter, and in other - the antimatter. In whole, in alternation of the worlds from the matter and antimatter the charge symmetry also can be restored in the scales of the extended cosmological space of the Big Universe. 


\section{Conclusion}

Thus, at consecutive following to GR and cosmological principle there are strong theoretical and observational reasons to represent the universe as a 3-sphere expanding (with slowing down) in the real 4-space.

At first, the Big Bang could occur only in the universe of finite mass and volume and a simplest (free on arbitrary hypotheses and parameters) consistent model of GR with such scenario is the closed model in 4-space, which at accounting of kinematic effects becomes exactly solvable and quite simple.

Secondly, the observed redshifts of SN 1a are well described by such model at realistic parameters of the Universe, relatively small fraction of dark matter and without hypothetical dark energy.

The observational discovery of additional cosmological redshift should be treated in the framework of GR as first indirect evidence of fourth spatial dimension, which has far-reaching and fundamental consequences both for cosmology and particle physics.

Thus, the new treatment of relativistic cosmology, developed in the previous [8] and present papers, almost uniquely follows from requirements of GR and principles of observational cosmology, is simple, solves the main cosmological problems and practically does not lead to new problems though puts many prospects for further research.

The further improvements of the model are related by taking into account of quantum effects and particle physics effects in superdense states, and also, at necessity, the effects of $4 \mathrm{~d}$-gravity. Since the relationship between the cosmological component of redshifts of objects with distances can be considered as known, the model allows one to study more adequately the proper evolution and spatial distribution of these distant sources.

\section{Appendix 1. The existence theorem for 3-sphere}

Theorem. For the existence of a $d$-sphere it is necessary and sufficient the embedding it in $(d+1)$-dimensional Riemannian space with flat or centrally symmetric metric around the $d$-sphere.

The proof of sufficiency is the standard procedure of constructing of $d$-spheres by embedding them into higher dimensional spaces.

The proof of the necessity [11] is based on the fact that a d-dimensional sphere topologically invariantly divides the embedding manifold on two disconnected (exterior and interior) regions. As the result, $d$-dimensional "area" $S_{d}$ of the $d$-sphere of radius $a$ is proportional to a $(d+1)$-dimensional volume $V_{d+1}$ of interior region of the sphere. From the expressions:

$$
\begin{gathered}
S_{d}=\int_{0}^{a} \delta(a-r) r^{d} d r \int d^{d-1} \Omega=a^{d} \int d^{d-1} \Omega, \\
V_{d+1}=\int_{0}^{a} r^{d} d r \int d^{d-1} \Omega=\frac{a^{d+1}}{d+1} \int d^{d-1} \Omega,
\end{gathered}
$$

follows

$$
S_{d}=\frac{d+1}{a} \cdot V_{d+1} .
$$

If the $d$-sphere does not embedded in $d+1$-dimensional space, then the volume of the interior region is equal to zero $V_{d+1}=0$, but then according to (83) the $d$-dimensional 
"area" of the sphere $S_{d}=0$ vanishes too. Therefore for the existence of the $d$ dimensional sphere it is necessary to embed it (at least) in $d+1$-dimensional space.

For a circle as a one-dimensional sphere on a plane and for 2-sphere in 3-space the formula (83) is obvious, while for the 3 -sphere in 4 -space we have:

$$
S_{3}=2 \pi^{2} a^{2}=\frac{4}{a} \cdot \frac{\pi^{2}}{2} a^{4}=\frac{3+1}{a} \cdot V_{4} .
$$

Thus, the 3-sphere with non-zero "area" $S_{3}>0$ exists only at embedding it at least into a real 4-space, only at which there is an internal region of non-zero 4-volume $V_{4} \sim S_{3}$.

\section{Appendix 1. Einstein's equations for the closed model in terms of world time}

For 3-sphere in $D=1+3$ spacetime with the world time $t$ and 3 -spherical coordinates $x^{\mu}=(t, \chi, \theta, \varphi)$ the metric has the form (see also [9]):

$$
g_{\mu v}=\left(\begin{array}{cccc}
e^{v} & 0 & 0 & 0 \\
0 & -a^{2} & 0 & 0 \\
0 & 0 & -a^{2} \sin ^{2} \chi & 0 \\
0 & 0 & 0 & -a^{2} \sin ^{2} \chi \sin ^{2} \theta
\end{array}\right),
$$

where $g_{00}=e^{v}=1-\dot{a}^{2}$. The Christoffel symbols $\Gamma_{\mu \nu}^{\lambda}$ for this metric are equal to:

$$
\begin{aligned}
& \Gamma_{00}^{0}=\frac{\dot{v}}{2}=\frac{-\dot{a} \ddot{a}}{1-\dot{a}^{2}}, \quad \Gamma_{11}^{0}=a \dot{a} e^{-v}=\frac{a \dot{a}}{1-\dot{a}^{2}}, \quad \Gamma_{10}^{1}=\Gamma_{20}^{2}=\Gamma_{30}^{3}=\frac{\dot{a}}{a}, \\
& \Gamma_{22}^{0}=a \dot{a} e^{-v} \sin ^{2} \chi=\frac{a \dot{a}}{1-\dot{a}^{2}} \sin ^{2} \chi, \quad \Gamma_{33}^{1}=-\sin \chi \cos \chi \sin ^{2} \theta, \\
& \Gamma_{33}^{0}=a \dot{a} e^{-v} \sin ^{2} \chi \sin ^{2} \theta=\frac{a \dot{a}}{1-\dot{a}^{2}} \sin ^{2} \chi \sin ^{2} \theta, \Gamma_{22}^{1}=-\sin \chi \cos \chi, \\
& \Gamma_{12}^{2}=\Gamma_{13}^{3}=\Gamma_{23}^{3}=\frac{\cos \chi}{\sin \chi}, \Gamma_{33}^{2}=-\sin \theta \cos \theta .
\end{aligned}
$$

The Ricci tensor components $R_{\mu \nu}=g^{\lambda \gamma} R_{\lambda \mu \gamma v}$ and the Ricci scalar $R=g^{\mu v} R_{\mu \nu}$ then take the form:

$$
\begin{aligned}
& R_{00}=-\frac{3}{a}\left(\ddot{a}-\frac{1}{2} \dot{a} \dot{v}\right)=-\frac{3 \ddot{a}}{a\left(1-\dot{a}^{2}\right)}, R_{22}=R_{11} \sin ^{2} \chi, \quad R_{33}=R_{22} \sin ^{2} \theta, \\
& R_{11}=-\left(\frac{1}{2} a \dot{a} \dot{v}-a \ddot{a}-2 \dot{a}^{2}\right) e^{-v}+2=\frac{a \ddot{a}}{\left(1-\dot{a}^{2}\right)^{2}}+\frac{2}{1-\dot{a}^{2}}, \\
& R=-\frac{6}{a^{2}}\left(\frac{1}{2} a \dot{a} \dot{v}-a \ddot{a}-\dot{a}^{2}-e^{v}\right) e^{-v}=-\frac{6}{a^{2}\left(1-\dot{a}^{2}\right)}-\frac{6 \ddot{a}}{a\left(1-\dot{a}^{2}\right)^{2}} .
\end{aligned}
$$

For comoving to 3 -sphere matter with 4 -velocity $u^{\mu}=\left[\left(1-\dot{a}^{2}\right)^{-1 / 2}, 0,0,0\right)$, the energy density $\rho$ and the pressure $p$ components of the energy-momentum tensor $T_{v}^{\mu}=(\rho+p) u^{\mu} u_{v}-p \delta_{v}^{\mu}$ are equal to:

$$
T_{0}^{0}=\rho, T_{1}^{1}=T_{2}^{2}=T_{3}^{3}=-p .
$$

Einstein's equations 


$$
G_{v}^{\mu}=R_{v}^{\mu}-\frac{1}{2} \delta_{v}^{\mu} R=\kappa_{4} T_{v}^{\mu}
$$

in components give the equations for evolution of radius, matter density and pressure:

$$
\begin{gathered}
G_{0}^{0}=\frac{3}{a^{2}}\left(1+\frac{\dot{a}^{2}}{e^{v}}\right)=\kappa_{4}\left[(\rho+p) \frac{e^{v}}{1-\dot{a}^{2}}-p\right], \\
G_{1}^{1}=\left(\ddot{a}-\frac{\dot{a} \dot{v}}{2}-\frac{\dot{a}^{2}}{a}\right) \frac{e^{-v}}{a}+\frac{1}{a^{2}}=-\kappa_{4} p .
\end{gathered}
$$

At the metric $e^{v}=1-\dot{a}^{2}$ from the linear element (9) for 3-sphere expanding in 4-space, these equations take a quite simple form [9]:

$$
\begin{gathered}
G_{0}^{0}=\frac{3}{a^{2}} \frac{1}{1-\dot{a}^{2}}=\kappa_{4} \rho, \\
G_{1}^{1}=\frac{2 \ddot{a}}{a\left(1-\dot{a}^{2}\right)^{2}}+\frac{1}{a^{2}\left(1-\dot{a}^{2}\right)}=-\kappa_{4} p
\end{gathered}
$$

In usual applications of the gravity theory in 3-space the constant $\kappa_{3}$ has been defined so that for a spherically-symmetric source a solution at large distances led to Newton's law in flat space, from which it has been obtained:

$$
\kappa_{3}=4 \pi \cdot 2 G
$$

where $G$ is a gravitational constant. However, when a spatial geometry for homogeneous matter distribution with a constant curvature corresponds to the 3-sphere in 4-space, the "asymptotically flat" are not the distant, but only the local regions only. For this reason the constant $\kappa_{4}$ in (92) should be defined by appropriately taking into account non-trivial topology of the space and we will perform that below.

To find the constant $\kappa_{4}$, let us consider the uniformly distributed matter in a small ball, when in the expression for $a_{m}$ (12) we can use the former $3 \mathrm{~d}$ expression (94) for $\kappa_{3}$. Then the result we will represent in a dependent on geometry form, which could be applied to regions of arbitrary large volume:

$$
\tilde{a}_{m}=2 G \cdot \frac{4 \pi}{3} \tilde{a}^{3} \rho_{0}=2 G M, \quad M=\frac{4 \pi}{3} \tilde{a}^{3} \rho_{0} .
$$

Here, instead of the local density $\rho_{0}$ and the 3 -volume of the ball a radius $\tilde{a}$, we directly express $\tilde{a}_{m}$ through the total mass-energy $M$ of matter in this volume. Thus, the maximal radius of expansion of such ball $a_{m}$ formally corresponds to its "gravitational radius".

For the Universe as a whole now we reverse this procedure - for $a_{m}$ we start with the expression for "the gravitational radius" with the mass-energy of the Universe $M$, and then express this total mass through the local density in 3-sphere:

$$
\begin{aligned}
& a_{m}=2 G M=2 G \cdot 2 \pi^{2} a_{0}^{3} \cdot \rho_{0}=4 \pi^{2} G \cdot a_{0}^{3} \rho_{0}, \\
& M=2 \pi^{2} a_{0}^{3} \cdot \rho_{0} .
\end{aligned}
$$

As the result we obtain the required expression for $\kappa_{4}$ :

$$
\kappa_{4}=6 \pi^{2} \cdot 2 G=12 \pi^{2} G .
$$




\section{References}

1. Einstein A. (1917) Sitz. Preus. Akad. Wiss. 1, 142.

2. Friedmann A. (1922) Zs. Phys. 11, 377.

3. Lemaître G. (1927) Ann. Soc. Sci. Brux., 47A, 49.

4. Hubble E. (1929) Proc. Nat. Acad. Sci. 15 (3), 168

5. Milne E. A. (1934) Quart. J. Math. Oxf. 5, 64.

6. Lemaître G. (1933) Ann. Soc. Sci. Brux., 53A, 51.

7. Gamov G. (1946) Phys. Rev. 70, 572.

8. Zakir Z. (2013) Theor. Phys. Astroph.\& Cosmol. 8(2) 24.

9. Park Ch.-G. (2007) arXiv:0710.1700.

10. Riess A. G. et al. (1998) Astron. J. 116, 1009;

Perlmutter S. et al. (1999) Astrophys. J. 517, 565.

11. Zakir Z. (2006) Theor. Phys. Astroph.\& Cosmol, 1(3) 42.

12. Zakir Z. (2013) Theor. Phys. Astroph.\& Cosmol. 8(1), 1; 8(1), 7.

13. Zakir Z. (2013) Theor. Phys. Astroph.\& Cosmol. 8(1), 16. 